\title{
Hypoalbuminemia and Hyperalbuminuria as Major Factors Regulating Circadian Blood Pressure Rhythm in Normal Subjects and Non-diabetic Patients with Early Stages of Chronic Kidney Disease
}

\author{
Gen Yasuda*, Daisaku Ando and Nobuhito Hirawa
}

Division of Nephrology and Hypertension, Yokohama City University Center Hospital, Kanagawa, Japan

\begin{abstract}
Study background: Albuminuria and nighttime blood pressure (BP) elevation are notable risk markers of chronic kidney disease (CKD). In non-diabetic patients with albuminuria, the effect of albuminuria on nocturnal BP and other risk factors that may disrupt circadian BP rhythm remain unclear. The aim of this study was to evaluate the relationship among albuminuria, serum albumin level and 24-hour BP rhythm in non-diabetic subjects.
\end{abstract}

Methods: This prospective study enrolled 778 subjects (aged 20-80 years, estimated glomerular filtration rate $\geq$ $60 \mathrm{~mL} / \mathrm{min} / 1.73 \mathrm{~m}^{2}$ ). Main outcome measure was sleeping/waking mean BP (MBP) ratio as an indicator of circadian BP rhythm. First, a cross-sectional study was performed in all individuals showing no proteinuria. Second, a study was done in subjects never treated for hypertension (hypertensive-naïve) stratified by urinary albumin excretion. Third, an intervention study was conducted in patients with massive albuminuria and hypoalbuminemia to examine circadian BP changes induced by treatment

Results: In 441 subjects without proteinuria, multivariate regression analyses identified serum albumin, cholestero level and circadian sympathovagal rhythm as independent predictors of sleeping/waking MBP ratio. Among hypertensivenaïve non-diabetic subjects $(n=231$ ), sleeping/waking MBP ratio increased with increasing albuminuria and decreasing serum albumin. Of 37 non-diabetic nephrotic patients who underwent treatment, 25 with serum albumin level increased to $>3 \mathrm{~g} / \mathrm{dL}$ showed a significant decrease $(P=0.026)$ in sleeping/waking MBP ratio after treatment, whereas 13 with urinary albumin/creatinine lowered to $<300 \mathrm{mg} / \mathrm{g}$ showed no significant difference $(P=0.191)$.

Conclusions: This study identified low serum albumin as an independent predictor of loss of nocturnal BP decline, which was also associated with massive albuminuria. Furthermore, sleeping/waking MBP ratio was improved after hyperalbuminuria and hypoalbuminemia were reversed by treatment. These findings suggest that low serum albumin level and massive albuminuria are risk markers of disrupted circadian BP rhythm in non-diabetic early stage CKD.

Keywords: Ambulatory blood pressure; Autonomic nervous activity; Diabetic nephropathy; Glomerulonephritis; Proteinuria

\section{Introduction}

Increase in urinary albumin excretion and nighttime blood pressure (BP) elevation are notable risk markers of cardiovascular mortality and morbidity as well as end-stage chronic kidney disease (CKD). A large number of recent studies have focused on the relation between microalbuminuria and loss of nocturnal BP decline. This association is mostly recognized in patients with diabetic nephropathy $[1,2]$ and usually complicated by autonomic nervous dysfunction [3-5]. Thus, urinary albumin excretion and imbalance in circadian autonomic nervous activities are suspected to be the major factors involved in loss of nocturnal BP fall. A similar relationship has been reported in nondiabetic hypertensive patients with microalbuminuria [6,7]. However, whether albuminuria is a cause or consequence of abnormal nocturnal $\mathrm{BP}$ change and whether other risk factors are involved in impaired circadian BP rhythm remain to be elucidated.

In patients with non-diabetic nephropathy or glomerulonephritis, serum albumin concentrations always change in proportion to urinary albumin levels, showing an inverse relationship. Thus, if albuminuria occurs concomitantly with loss of nighttime BP fall, hypoalbuminemia is always present. However, few studies have focused on the influence of serum albumin level on the circadian BP rhythm in patients with no major traditional risk factors such as diabetes, hypertension and advanced renal failure. Besides, many studies evaluated mainly the relationship between microalbuminuria and $24-\mathrm{h} \mathrm{BP}$ rhythm by treating hypertension and diabetes to eliminate major risk factors [8-11]. No studies so far have assessed how direct treatment of albuminuria and hypoalbuminemia influences $24-\mathrm{h}$ BP rhythm, because it is difficult to resolve albuminuria in patients with primary glomerulonephritis. Whether vigorous changes in urinary and serum albumin levels through treatment affect the circadian BP rhythm can only be evaluated in patients with massive albuminuria but no traditional risk factors.

The aim of the present study was to evaluate, first, the relationship between serum albumin levels and sleeping to waking BP ratios in subjects without proteinuria or advanced renal dysfunction; second, the relations among serum and urinary albumin levels and sleeping/ waking BP ratio in treatment-naïve subjects with various degrees of albuminuria; and third, how improvements of hypoalbuminemia and massive albuminuria influence circadian BP patterns.

*Corresponding author: Gen Yasuda, Division of Nephrology and Hypertension, Yokohama City University Center Hospital, 4-57, Urafune, Minami, Yokohama 232-0024, Kanagawa, Japan, Tel: +81 45261 5656; E-mail: y911429@yokohama-cu.ac.jp

Received January 21, 2014; Accepted February 21, 2014; Published February 24, 2014

Citation: Yasuda G, Ando D, Hirawa N (2014) Hypoalbuminemia and Hyperalbuminuria as Major Factors Regulating Circadian Blood Pressure Rhythm in Normal Subjects and Non-diabetic Patients with Early Stages of Chronic Kidney Disease. J Hypertens 3: 142. doi:10.4172/2167-1095.1000142

Copyright: (c) 2014 Yasuda G, et al. This is an open-access article distributed under the terms of the Creative Commons Attribution License, which permits unrestricted use, distribution, and reproduction in any medium, provided the original author and source are credited. 
Citation: Yasuda G, Ando D, Hirawa N (2014) Hypoalbuminemia and Hyperalbuminuria as Major Factors Regulating Circadian Blood Pressure Rhythm in Normal Subjects and Non-diabetic Patients with Early Stages of Chronic Kidney Disease. J Hypertens 3: 142. doi:10.4172/21671095.1000142

Page 2 of 7

\section{Methods}

\section{Patients}

Subjects aged between 20 and 80 years who were referred to our hospital between January 1, 2000 and December 31, 2012 because of abnormal urinalysis found at primary care physicians' offices or regular medical check-ups were enrolled in the study. Participants were eligible for the study if they had estimated glomerular filtration rate (eGFR) $\geq$ $60 \mathrm{~mL} / \mathrm{min} / 1.73 \mathrm{~m} 2$. Patients were excluded if they met the following criteria: malnutrition; protein-losing enteropathy; polycystic kidney disease; tubulointerstitial nephritis; endocrinological, hematological or hepatic disease; uncontrolled hypertension ( $>160 \mathrm{~mm} \mathrm{Hg}$ in systolic $\mathrm{BP}$ or $>100 \mathrm{~mm} \mathrm{Hg}$ in diastolic BP); abnormal thyroid function; other major diseases. Informed consent was obtained from each subject. Detailed medical history, demographic characteristics, and anthropometric measurements were obtained from every subject. This study was approved by the Ethics Committee of the Yokohama City University Center Hospital.

\section{Study design}

This prospective research consists of three studies to compare sleeping/waking BP ratios obtained by ambulatory BP monitoring (ABPM). The first was a cross-sectional study in all individuals who had a negative dipstick test for proteinuria. The second was a study in subjects who had never received treatment for hypertension (hypertensivenaïve) stratified into four subgroups by urinary albumin excretion (UAE). The third was an intervention study with intention-to treat analysis in patients with massive albuminuria and hypoalbuminemia to examine the changes in circadian BP rhythm induced by treatment. In each study, blood samples for biochemical parameters and spot urine sample for UAE were collected when ABPM was performed. All subjects underwent $\mathrm{ABPM}$ using a device equipped with power spectral analysis of heart rate variability (TM2425, A \& D, Tokyo, Japan). The methods of measurement have been described in detail elsewhere [12]. Mean BP (MBP) was calculated as the sum of diastolic BP and one-third of pulse pressure, for the waking and sleeping periods separately. Then, sleeping/ waking MBP ratio was analyzed as an index of the circadian BP rhythm. The power spectral densities of the heart rate variability were divided into two components: a low frequency (LF) component $(0.05-0.15 \mathrm{~Hz})$, and a high frequency (HF) component (0.15-0.4 Hz). Sleeping/waking ratio of the $\mathrm{LF} / \mathrm{HF}$ ratio (sleeping/waking $\mathrm{LF} / \mathrm{HF}$ ratio) was analyzed to evaluate the circadian rhythm of sympathovagal nervous activities.

\section{Study 1: Predictors for increased sleeping/waking MBP ratio in subjects without proteinuria}

To analyze the clinical factors other than albuminuria which independently affect sleeping/waking MBP ratio, all patients with a negative dipstick test for proteinuria (no microalbuminuria) were selected among the enrolled subjects. Those who showed readings of trace or above in urine dipstick tests were excluded. Urine dipsticks were analyzed by an automated device capable of reading color changes of the strips to detect proteinuria. In a preliminary analysis, among 650 subjects who had a negative dipstick test, 551 (85\%) showed urinary albumen excretion lower than $30 \mathrm{mg} / \mathrm{g}$ creatinine determined simultaneously by a turbidimetric immunoassay kit (Mitsubishi Chemical, Tokyo). Thus, a negative dipstick test for proteinuria indicated urinary protein levels lower than the cut-off for microalbuminuria of $30 \mathrm{mg} / \mathrm{g}$ creatinine. In all subjects, ABPM and blood and urine tests were conducted as described above. Multivariate regression analyses were performed to identify factors affecting circadian BP rhythm.
The multivariate model consisted of sleeping/waking MBP ratio as the dependent variable and other potential confounding predictors as independent variables, including age, gender, body mass index, diabetic or non-diabetic, hemoglobin, hemoglobin A1C, serum albumin, total cholesterol, triglycerides, eGFR, 24-h MBP, sleeping/waking LF/HF ratio, alcohol consumption, smoking, and the number of hypertensive drugs. Diabetes was defined as hemoglobin A1C higher than $6.5 \%$ or a positive oral glucose tolerance test. Smoking and alcohol consumption were classified as never versus current or past.

\section{Study 2: Comparison of sleeping/waking MBP ratio with other parameters between diabetic and non-diabetic patients among hypertensive-naïve subjects}

To control for the effect of anti-hypertensive medications, hypertensive-naïve patients were selected among the enrolled subjects. Furthermore, to control for the risk factor of diabetes, the hypertensivenaïve subjects were divided into diabetic and non-diabetic groups. Then both groups were further stratified into four subgroups based on UAE: normoalbuminuria group with UAE $<30 \mathrm{mg} / \mathrm{g}$ creatinine, microalbuminuria group with $30 \mathrm{mg} / \mathrm{g} \leq \mathrm{UAE}<300 \mathrm{mg} / \mathrm{g}$ creatinine, subnephrotic group with $300 \mathrm{mg} / \mathrm{g} \leq \mathrm{UAE}<3,000 \mathrm{mg} / \mathrm{g}$ creatinine, and nephrotic group with $\mathrm{UAE} \geq 3,000 \mathrm{mg} / \mathrm{g}$ creatinine. In all subjects, ABPM and blood and urine tests were conducted as described above. Sleeping/waking MBP ratio, other parameters obtained from ABPM and clinical characteristics were compared among the subgroups. The relations among serum and urinary albumin levels and sleeping/waking $\mathrm{BP}$ ratio were analyzed.

\section{Study 3: Changes in sleeping/waking MBP ratio and other parameters after treatment in non-diabetic nephrotic patients}

To examine the effect of immunosuppressive therapy for hypoalbuminemia and albuminuria on sleeping/waking MBP ratio, 37 hypertensive-naïve, non-diabetic, nephrotic patients identified in study 2 were selected. ABPM together with blood and urine tests were conducted before treatment. The patients initially received intravenous methylprednisolone $(0.5$ or $1.0 \mathrm{~g} /$ day for three days $)$ followed by combination therapy with oral cyclosporine ( 2 to $3 \mathrm{mg} / \mathrm{kg} / \mathrm{day}$ ) and prednisolone (30 to $40 \mathrm{mg} /$ day) or prednisolone monotherapy. The prednisolone and cyclosporine doses were tapered by 5-10 mg and 25 mg, respectively, every 4-8 weeks according to the urinary albumin excretion volume. Medications were ceased when remission was confirmed clinically. Patients were followed for 24 months from the start of therapy. All patients underwent the second ABPM between 6 and 24 months, regardless of whether or not they had remission. Remission was defined as a decrease in UAE to $<300 \mathrm{mg} / \mathrm{g}$ creatinine or an increase in serum albumin level to $>3.0 \mathrm{~g} / \mathrm{dL}$ on two consecutive visits. To avoid false results caused by fluctuations in albuminuria, remission had to persist at least one month. Subjects who did not fulfill the criteria of remission were classified into the non-remission group. Patients treated with antihypertensive agents and diuretics prior to the second ABPM suspended the medications for at least two weeks before ABPM.

\section{Statistical Analysis}

Normally distributed parameters are presented as means \pm standard error of the mean (SEM). Skewed distribution was normalized by logarithmic transformation before analyses. Based on previous studies $[11,13-15]$, it was estimated that therapy would induce a difference in logarithm UAE of 0.4 or more with standard deviation of $0.8 \mathrm{mg} / \mathrm{g}$ creatinine. Then after adjustment for possible withdrawals and non- 
Citation: Yasuda G, Ando D, Hirawa N (2014) Hypoalbuminemia and Hyperalbuminuria as Major Factors Regulating Circadian Blood Pressure Rhythm in Normal Subjects and Non-diabetic Patients with Early Stages of Chronic Kidney Disease. J Hypertens 3: 142. doi:10.4172/21671095.1000142

Page 3 of 7

compliance, the intention-to-treat effect was calculated to be $5-10 \%$ risk reduction. A minimum sample size of 36 cases was required to provide $80 \%$ power with a type error of $5 \%$. Categorical variables were analyzed by $\chi^{2}$ test. The differences in baseline parameters between two groups were assessed by the nonparametric Mann-Whitney's $U$ test. Data obtained before and after therapy were analyzed using the Wilcoxon signed-rank test. The significance of differences among four subgroups was tested by one-way analysis of variance (ANOVA) and the Bonferroni's method. Multivariate regression analysis was used to determine the contribution of the variables to the sleeping/waking $\mathrm{BP}$ ratio. Variables associated by univariate analysis were included in the multivariate model. A P value less than 0.05 was considered statistically significant. Statistical analyses were performed using SPSS version 20 (SPSS, Chicago, IL, USA).

\section{Results}

\section{Predictors of increase in sleeping/waking MBP ratio in} subjects without proteinuria

Among 778 subjects enrolled, 441 had a negative dipstick test for proteinuria (reading below "trace"). The clinical parameters of these subjects are summarized in Table 1. Multivariate regression analyses were conducted to examine the clinical parameters (independent variables) associated with sleeping/waking MBP ratio (dependent variable). The analyses identified serum albumin level as an independent predictor that correlated inversely with sleeping/waking MBP ratio. Sleeping/waking LF/HF ratio and serum total cholesterol level were also identified as independent predictors of sleeping/waking MBP ratio.

Comparison of sleeping/waking MBP ratio and other parameters between diabetic and non-diabetic patients in hypertensive-naïve subjects

All hypertensive-naïve subjects $(n=297)$ were classified into a non-diabetic and a diabetic group, and further stratified into four subgroups based on UAE. Clinical parameters of the subgroups are summarized in Table 2. No significant differences in gender, body mass index, serum triglycerides and eGFR were detected among four subgroups both in diabetic and non-diabetic groups. All non-diabetic subgroups except for the nephrotic subgroup were younger than their counterparts in the diabetic group. The normoalbuminuria subgroup was the oldest among the non-diabetic subgroups. In both diabetic and non-diabetic groups, serum albumin level was not significantly different between the normoalbuminuria and microalbuminuria subgroups but was significantly lower in the nephrotic group. Serum total cholesterol level and urinary albumin excretion increased in the order of microalbuminuria, subnephrotic and nephrotic subgroup in both groups. Twenty-four-hour MBP was not significantly different among four subgroups in both diabetic and non-diabetic groups (Figure 1). However, the three albuminuria-positive diabetic subgroups had significantly higher 24-h MBP than their counterparts in the nondiabetic group. The sleeping/waking MBP ratio increased significantly with increasing severity of albuminuria in the non-diabetic group (Figure 2). In the diabetic group, the sleeping/waking MBP ratio did not differ significantly among the four subgroups, but the ratios were higher than their counterparts in the non-diabetic group except for the nephrotic subgroup. Sleeping/waking LF/HF ratios did not differ significantly among four subgroups in both diabetic and non-diabetic groups. However, the normoalbuminuria and microalbuminuria subgroups in the non-diabetic group showed significantly lower ratios than their counterparts in the diabetic group (Figure 3).

\section{Changes in sleeping/waking MBP ratio and other parameters after treatment in the non-diabetic nephrotic subgroup}

Thirty-seven non-diabetic nephrotic patients underwent ABPM before and after immunosuppressive therapy. The pathological findings in these patients were minimal change $(n=17)$, membranoproliferative glomerulonephritis $(n=3)$, membranous nephropathy ( $n=9)$, lupus nephritis $(\mathrm{n}=5)$, mesangial proliferative glomerulonephritis $(\mathrm{n}=1)$, and IgA nephropathy $(\mathrm{n}=2)$. The changes in clinical parameters from before to after treatment in the remission and non-remission groups are shown in Tables 3 and 4 . When the criterion of remission was UAE lower than $300 \mathrm{mg} / \mathrm{g}$ creatinine, 13 patients entered complete remission. In these patients, sleeping/waking MBP ratio did not change significantly, although serum albumin level increased and UAE decreased after remission compared to before remission (Table 3). When serum albumin level higher than $3 \mathrm{~g} / \mathrm{dL}$ was used as the criterion, however, 25 patients entered remission with a significant decrease in sleeping/waking MBP ratio (Table 4). The maintenance doses of prednisolone in the remission and non-remission groups were respectively $2.8 \pm 4.0$ and $3.3 \pm 4.3 \mathrm{mg} /$ day $(P=0.584)$ using the criterion

\begin{tabular}{|c|c|c|c|c|c|}
\hline Variables & Participants $(n=441)$ & $\beta$ coefficient & Standardized $\beta$ coefficient & Standard error & $P$ \\
\hline Age & $57 \pm 1$ & $<0.001$ & 0.092 & 0.001 & 0.610 \\
\hline Gender (men/women) & $158 / 283$ & -0.061 & -0.080 & 0.061 & 0.316 \\
\hline Body mass index $\left(\mathrm{kg} / \mathrm{m}^{2}\right)$ & $23.5 \pm 0.3$ & -0.003 & -0.140 & 0.002 & 0.191 \\
\hline Diabetes (diabetes/non-diabetes) & $72 / 369$ & 0.017 & 0.111 & 0.020 & 0.392 \\
\hline Hemoglobin (g/dL) & $13.6 \pm 0.1$ & -0.006 & -0.121 & 0.006 & 0.305 \\
\hline Hemoglobin A1C (\%) & $5.8 \pm 0.1$ & 0.002 & 0.045 & 0.006 & 0.709 \\
\hline Albumin (g/dL) & $4.4 \pm 0.1$ & -0.050 & -0.239 & 0.024 & 0.020 \\
\hline Total cholesterol (mg/dL) & $207 \pm 2$ & $<0.001$ & 0.172 & $<0.001$ & 0.047 \\
\hline Triglycerides (mg/dL) & $139 \pm 4$ & $<0.001$ & -0.023 & $<0.001$ & 0.829 \\
\hline eGFR $\left(\mathrm{ml} / \mathrm{min} / 1.73 \mathrm{~m}^{2}\right)$ & $79 \pm 1$ & -0.001 & -0.117 & 0.002 & 0.775 \\
\hline 24-hour mean blood pressure $(\mathrm{mm} \mathrm{Hg})$ & $102 \pm 1$ & $<0.001$ & 0.019 & $<0.001$ & 0.731 \\
\hline Sleeping/waking LF/HF ratio (\%) & $79 \pm 1$ & 0.049 & 0.162 & 0.022 & 0.023 \\
\hline Alcohol (never/current/past, \%) & $45 / 42 / 13$ & 0.015 & 0.147 & 0.015 & 0.345 \\
\hline Smoking (never/current /past, \%) & $38 / 39 / 23$ & 0.002 & 0.019 & 0.015 & 0.905 \\
\hline Number of hypertensive medications used (none/one/two/three or more) & $196 / 104 / 97 / 44$ & -0.008 & -0.134 & 0.008 & 0.332 \\
\hline
\end{tabular}

Model summary, adjusted $\mathrm{R}^{2}=0.249, P<0.001$. eGFR, estimated glomerular filtration rate; HF, high frequency component; LF, low frequency component. Body mass index was calculated by the formula; body weight $(\mathrm{kg}) / \mathrm{height}(\mathrm{m})^{2}$

Table 1: Multivariate regression analyses of background clinical parameters associated with sleeping/waking ratio of mean blood pressure. 
Citation: Yasuda G, Ando D, Hirawa N (2014) Hypoalbuminemia and Hyperalbuminuria as Major Factors Regulating Circadian Blood Pressure Rhythm in Normal Subjects and Non-diabetic Patients with Early Stages of Chronic Kidney Disease. J Hypertens 3: 142. doi:10.4172/21671095.1000142

Page 4 of 7

\begin{tabular}{|c|c|c|c|c|c|}
\hline Parameter & Normoalbuminuria & Microalbuminuria & Subnephrotic & Nephrotic & ANOVA \\
\hline \multicolumn{6}{|l|}{ Non-diabetic group } \\
\hline Number & 91 & 37 & 66 & 37 & \\
\hline Age (years) & $53 \pm 2$ & $42 \pm 2^{\mathrm{a}}$ & $42 \pm 2^{\mathrm{a}}$ & $44 \pm 3^{a}$ & $<0.001$ \\
\hline Gender (men/women) & $31 / 60$ & $15 / 22$ & $30 / 35$ & $19 / 18$ & 0.179 \\
\hline Body mass index $\left(\mathrm{kg} / \mathrm{m}^{2}\right)$ & $22.5 \pm 0.4$ & $22.2 \pm 0.8$ & $23.8 \pm 0.5$ & $22.3 \pm 0.6$ & 0.060 \\
\hline Serum albumin (g/dL) & $4.5 \pm 0.1$ & $4.3 \pm 0.1$ & $3.9 \pm 0.1^{\mathrm{ab}}$ & $1.8 \pm 0.2^{\mathrm{abc}}$ & $<0.001$ \\
\hline Total cholesterol (mg/dL) & $211 \pm 4$ & $191 \pm 9$ & $230 \pm 10$ & $402 \pm 29^{a b c}$ & $<0.001$ \\
\hline Triglycerides (mg/dL) & $136 \pm 17$ & $126 \pm 17$ & $158 \pm 11$ & $173 \pm 16$ & 0.327 \\
\hline eGFR (mL/min/1.73m²) & $78 \pm 2$ & $82 \pm 3$ & $79 \pm 2$ & $80 \pm 3$ & 0.637 \\
\hline Log urinary albumin excretion (mg/g creatinine) & ND & $2.0 \pm 0.1$ & $2.9 \pm 0.1 b$ & $3.9 \pm 0.1^{\mathrm{bc}}$ & $<0.001$ \\
\hline \multicolumn{6}{|l|}{ Diabetic group } \\
\hline Number & 23 & 16 & 17 & 10 & \\
\hline Age (years) & $61 \pm 3^{d}$ & $61 \pm 3^{e}$ & $58 \pm 4^{e}$ & $54 \pm 3$ & 0.460 \\
\hline Gender (men/women) & $9 / 14$ & $9 / 7$ & $8 / 9$ & $4 / 6$ & 0.738 \\
\hline Body mass index $\left(\mathrm{kg} / \mathrm{m}^{2}\right)$ & $21.5 \pm 0.9$ & $24.8 \pm 1.7$ & $21.8 \pm 0.9$ & $23.6 \pm 1.5$ & 0.214 \\
\hline Serum albumin $(\mathrm{g} / \mathrm{dL})$ & $4.3 \pm 0.1$ & $4.3 \pm 0.1$ & $4.1 \pm 0.1$ & $2.7 \pm 0.3^{\text {abce }}$ & $<0.001$ \\
\hline Total cholesterol (mg/dL) & $222 \pm 10$ & $202 \pm 8$ & $229 \pm 12$ & $289 \pm 25^{d}$ & 0.070 \\
\hline Triglycerides (mg/dL) & $186 \pm 37$ & $193 \pm 42$ & $167 \pm 32$ & $206 \pm 36$ & 0.915 \\
\hline eGFR (mL/min/1.73m²) & $74 \pm 7$ & $81 \pm 7$ & $85 \pm 9$ & $75 \pm 12$ & 0.382 \\
\hline Log urinary albumin excretion (mg/g creatinine) & ND & $2.2 \pm 0.1$ & $2.7 \pm 0.3 b$ & $3.6 \pm 0.4^{\mathrm{bc}}$ & $<0.001$ \\
\hline
\end{tabular}

Data are expressed as mean \pm SEM or number of patients unless noted otherwise. eGFR, estimated glomerular filtration rate; ND, not detected. ${ }^{2} P<0.01$ vs. normoalbuminuria group; ${ }^{b} P<0.01$ vs. microalbuminuria group; ${ }^{c} P<0.01$ vs. subnephrotic group; ${ }^{d} P<0.05$, e $P<0.01$ vs. counterpart in the non-diabetes group

Table 2: Background clinical parameters in four subgroups stratified by urinary albumin excretion in non-diabetic and diabetic groups.

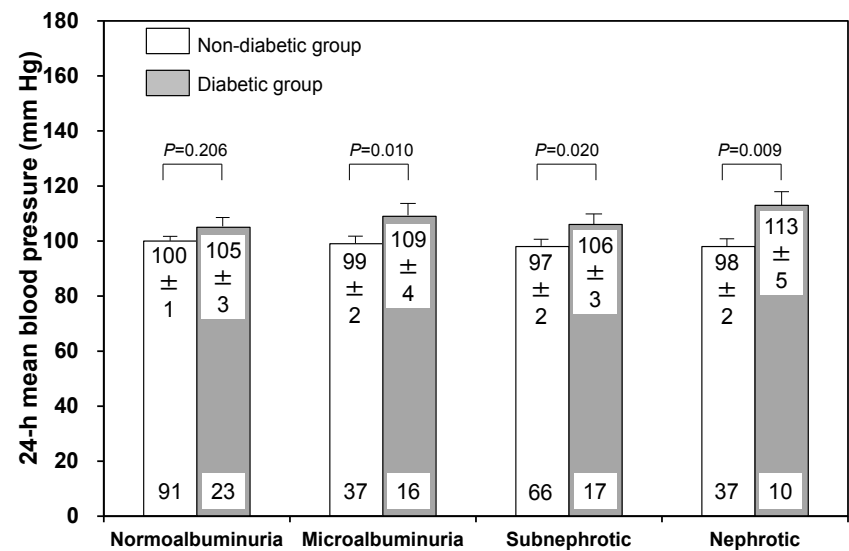

Figure 1: Twenty-four-hour mean blood pressure in the four subgroups stratified by urinary albumin excretion in the non-diabetic and diabetic groups. The figures at the top of each column are mean \pm SEM, and the figure at the bottom is the number of subjects. One-way analysis of variance (ANOVA) comparing the four subgroups yielded $P=0.311$ in the non-diabetic group and $P=0.411$ in the diabetic group.

of serum albumin concentrations $>3 \mathrm{~g} / \mathrm{dL}$, and were $4.0 \pm 4.6$ and $2.5 \pm$ $3.9 \mathrm{mg} /$ day using the criterion of UAE $<300 \mathrm{mg} / \mathrm{g}$ creatinine $(P=0.247)$, showing no differences with both criteria. No patients received other immunosuppressive medications at the second ABPM. There were no changes in 24-h MBP and eGFR after treatment compared to before treatment, regardless of treatment outcome.

\section{Discussion}

In this study, we demonstrated that the serum albumin was an independent predictor for loss of nocturnal BP decline in subjects who had no albuminuria or dipstick-positive proteinuria. The non-diabetic subjects showed no significant loss of nighttime BP fall until they had

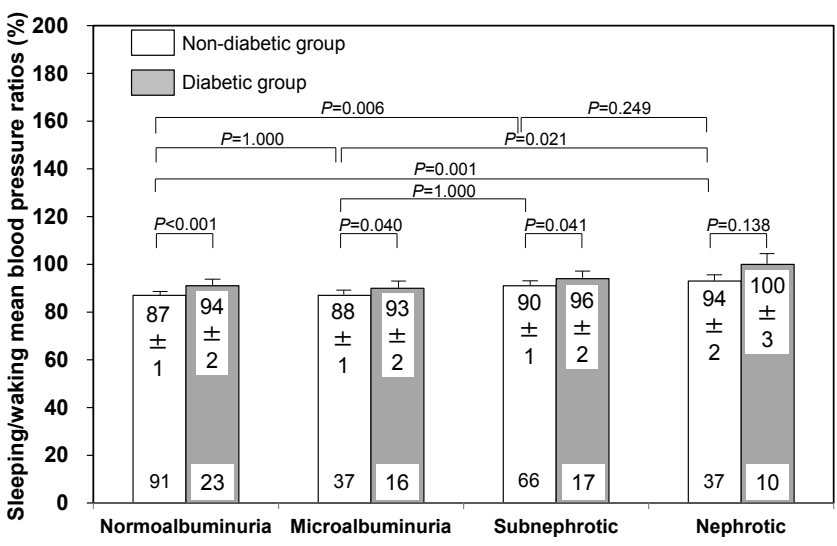

Figure 2: Sleeping/waking mean blood pressure ratios in the four subgroups stratified by urinary albumin excretion in the non-diabetic and diabetic groups. The figures at the top of each column are mean \pm SEM, and the figure at the bottom is the number of subjects. One-way analysis of variance (ANOVA) comparing the four subgroups yielded $P=0.310$ in the diabetic group and $P<0.001$ in the non-diabetic group.

massive albuminuria and hypoalbuminemia. Furthermore, in the nondiabetic nephrotic subgroup, improvements in hyperalbuminuria and hypoalbuminemia were associated with improvement in sleeping/ waking BP ratio. To the best of our knowledge, this is the first report that nighttime BP fall is associated with vigorous changes in urinary and serum albumin levels.

Microalbuminuria and loss of nighttime BP dip are the established risk markers of cardiovascular diseases, and often coexist in patients with diabetes or hypertension [16,17]. However, it remains controversial which marker acts first and affects the other. Some studies [18-21] reported the possibility that microalbuminuria influences circadian BP pattern and predicts cardiovascular disease mortality, 
Citation: Yasuda G, Ando D, Hirawa N (2014) Hypoalbuminemia and Hyperalbuminuria as Major Factors Regulating Circadian Blood Pressure Rhythm in Normal Subjects and Non-diabetic Patients with Early Stages of Chronic Kidney Disease. J Hypertens 3: 142. doi:10.4172/21671095.1000142

Page 5 of 7

but others $[1,3,8,22]$ indicated that disrupted 24 -h BP rhythm appears earlier than microalbuminuria. In our study, diabetic patients in the early stage of CKD showed loss of nighttime BP decline independent of UAE. These patients also showed imbalance in circadian autonomic nervous activities and increased 24-h MBP. These findings suggest that sustained exposure of organs and the entire vascular tree to elevated BP and hyperglycemia raises nighttime BP before damaging glomeruli to induce microalbuminuria. Thus, it is unlikely that microalbuminuria per se has predisposing pathophysiological effects to cause loss of nocturnal BP fall.

However, there still remains feasibility that preceding microalbuminuria causes loss of nighttime BP dipping in the early stage of CKD. Recently, microalbuminuria has been proposed to be a

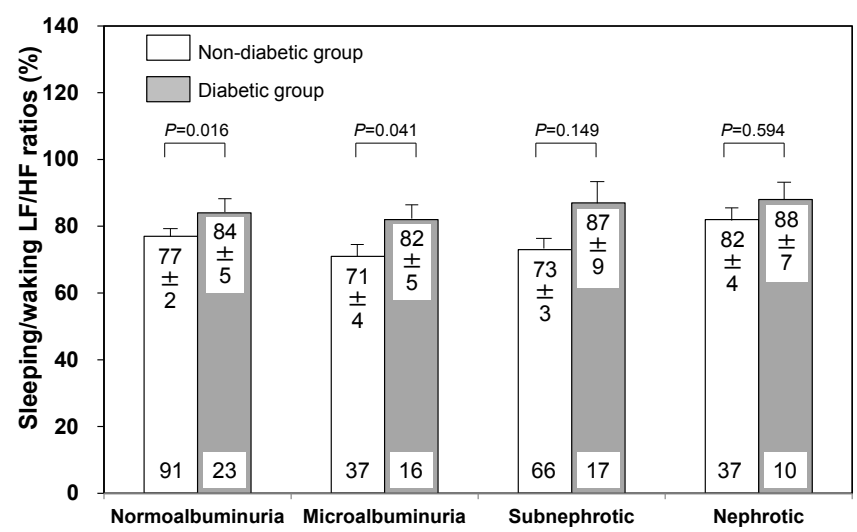

Figure 3: Sleeping/waking ratios of LF/HF ratio in the four subgroups stratified by urinary albumin excretion in the non-diabetic and diabetic groups. The figures at the top of each column are mean \pm SEM, and the figure at the bottom is the number of subjects. LF: low frequency; HF: high frequency. One-way analysis of variance (ANOVA) comparing the four subgroups yielded $P=0.121$ in the non-diabetic group and $P=0.201$ in the diabetic group. non-traditional risk factor of cardiovascular diseases independent of diabetes and hypertension [23]. Boulatov et al. [13] pointed out that elevated urinary albumin excretion already exists in the early stages of hypertension. Ärnlöv et al. [14] reported that urinary albumin excretion well below microalbuminuria level is a predictor of cardiovascular diseases even in non-hypertensive, non-diabetic individuals. However, Hoshide et al. [24] and Soylu et al. [25] showed that microalbuminuria causes no differences in the nighttime BP dip. Clausen et al. [26] also found normal nocturnal BP decline in normotensive subjects with elevated urinary albumin excretion. Our study showed that sleeping/waking BP ratio was not elevated in non-diabetic subjects with microalbuminuria. Taken together, these findings indicate that microalbuminuria does not directly elevate sleeping/waking BP ratio in early stage CKD at least in non-diabetic individuals. BP is regulated exclusively by cardiac and systemic vascular function, hemodynamic changes and neurohumoral factors. Thus, very low urinary albumin concentration does not seem to be powerful enough to directly cause nighttime BP elevation. We postulate that a greater burden of traditional cardiovascular risk factors persisting long-term would cause loss of nocturnal BP fall and elevate urinary albumin excretion.

Glomerulonephritis, an autoimmune disease, is another major kidney disease manifesting wide-range albuminuria as is observed in diabetic nephropathy, but the site of pathophysiological action is limited to the glomeruli. Systemic vascular damages are less severe in early stages of CKD than in diabetes and hypertension. Our data showed that in non-diabetic subjects including those with glomerulonephritis, the sleeping/waking MBP ratio did not increase until they had massive albuminuria. This indicates that nephrogenic range albuminuria and disturbed circadian BP variation are closely related. The nephrotic syndrome is a complex dysmetabolic disorder as well as an autoimmune disease manifesting increased interstitial fluids together with changes in circulating blood volume and humoral factors $[27,28]$. Thus, the BP

\begin{tabular}{|c|c|c|c|c|c|c|}
\hline \multirow[b]{2}{*}{ Parameter } & \multicolumn{2}{|c|}{ Remission } & \multirow[b]{2}{*}{$P$} & \multicolumn{2}{|c|}{ Non-remission } & \multirow[b]{2}{*}{$P$} \\
\hline & Before treatment & After treatment & & Before treatment & After treatment & \\
\hline Number of subjects & 13 & 13 & & 24 & 24 & \\
\hline 24-h MBP (mm Hg) & $94.4 \pm 2.4$ & $98.2 \pm 2.4$ & 0.433 & $99.7 \pm 2.4$ & $96.1 \pm 2.5$ & 0.077 \\
\hline Sleeping/waking MBP ratio & $0.94 \pm 0.02$ & $0.90 \pm 0.03$ & 0.191 & $0.95 \pm 0.02$ & $0.92 \pm 0.02$ & 0.086 \\
\hline Serum albumin (g/dL) & $1.7 \pm 0.1$ & $3.8 \pm 0.2$ & $<0.001$ & $1.8 \pm 0.2$ & $2.9 \pm 0.2$ & $<0.001$ \\
\hline Total cholesterol (mg/dL) & $465 \pm 46$ & $233 \pm 17$ & 0.018 & $366 \pm 33$ & $282 \pm 32$ & 0.028 \\
\hline eGFR $\left(\mathrm{mL} / \mathrm{min} / 1.73 \mathrm{~m}^{2}\right)$ & $77 \pm 5$ & $74 \pm 5$ & 0.363 & $81 \pm 5$ & $77 \pm 5$ & 0.581 \\
\hline Log urinary albumin excretion (mg/g creatinine) & $3.7 \pm 0.1$ & $1.7 \pm 0.1$ & $<0.001$ & $3.8 \pm 0.1$ & $3.4 \pm 0.1$ & 0.013 \\
\hline
\end{tabular}

Data are expressed as mean \pm SEM or number of patients unless noted otherwise. eGFR, estimated glomerular filtration rate; MBP, mean blood pressure

Table 3: Comparisons of clinical parameters before and after treatment in nephrotic subjects based on the remission criterion of urinary albumin excretion $<300 \mathrm{mg} / \mathrm{g}$ creatinine.

\begin{tabular}{|c|c|c|c|c|c|c|}
\hline \multirow[b]{2}{*}{ Parameter } & \multicolumn{2}{|c|}{ Remission } & \multirow[b]{2}{*}{$P$} & \multicolumn{2}{|c|}{ Non-remission } & \multirow[b]{2}{*}{$P$} \\
\hline & Before treatment & After treatment & & Before treatment & After treatment & \\
\hline Number of subjects & 25 & 25 & & 12 & 12 & \\
\hline 24-h MBP (mm Hg) & $97.4 \pm 2.3$ & $96.5 \pm 2.0$ & 0.458 & $97.8 \pm 2.5$ & $97.6 \pm 3.5$ & 0.594 \\
\hline Sleeping/waking MBP ratio & $0.95 \pm 0.02$ & $0.89 \pm 0.02$ & 0.026 & $0.97 \pm 0.02$ & $0.95 \pm 0.02$ & 0.328 \\
\hline Serum albumin $(\mathrm{g} / \mathrm{dL})$ & $1.8 \pm 0.1$ & $3.8 \pm 0.1$ & $<0.001$ & $1.7 \pm 0.2$ & $2.1 \pm 0.1$ & 0.092 \\
\hline Total cholesterol (mg/dL) & $407 \pm 39$ & $223 \pm 15$ & 0.005 & $393 \pm 38$ & $351 \pm 38$ & 0.080 \\
\hline eGFR $\left(\mathrm{mL} / \mathrm{min} / 1.73 \mathrm{~m}^{2}\right)$ & $80 \pm 4$ & $77 \pm 4$ & 0.265 & $77 \pm 8$ & $73 \pm 7$ & 0.929 \\
\hline Log urinary albumin excretion (mg/g creatinine) & $3.7 \pm 0.1$ & $2.3 \pm 0.2$ & $<0.001$ & $3.9 \pm 0.1$ & $3.7 \pm 0.2$ & 0.477 \\
\hline
\end{tabular}

Data are expressed as mean \pm SEM or number of patients unless noted otherwise. eGFR, estimated glomerular filtration rate; MBP, mean blood pressure

Table 4: Comparisons of clinical parameters before and after treatment in nephrotic subjects based on the remission criterion of serum albumin level $>3 \mathrm{~g} / \mathrm{dL}$. 
Citation: Yasuda G, Ando D, Hirawa N (2014) Hypoalbuminemia and Hyperalbuminuria as Major Factors Regulating Circadian Blood Pressure Rhythm in Normal Subjects and Non-diabetic Patients with Early Stages of Chronic Kidney Disease. J Hypertens 3: 142. doi:10.4172/21671095.1000142

Page 6 of 7

regulating mechanisms under these specific hemodynamic conditions should be different from those in minor albuminuria, resulting in loss of nighttime BP fall.

In the present study, we also evaluated the role of serum albumin level on the circadian BP rhythm, because hyperalbuminuria and hypoalbuminemia are inseparable signs and usually coexist. However, it is difficult to evaluate the relation between serum albumin concentration and circadian BP pattern without taking albuminuria into account. Only a few studies reported the association of serum albumin levels with less dipping BP [29]. Our study showed that even in cases of negative dipstick test for proteinuria or absence of microalbuminuria, serum albumin levels correlate inversely with nighttime BP fall. In nephrotic patients who achieved remission based on serum albumin levels by treatment, the sleeping/waking BP ratio improved as hypoalbuminemia and hyperalbuminuria were reversed. Recently low serum albumin level has also been considered to play a key role in systemic vascular damage. Yokoyama et al. [30] reported that hypoalbuminemia is an independent predictor of diabetic nephropathy and impaired renal function. Zimmermann et al. [31] reported a negative correlation between serum albumin level and C-reactive protein level, an indicator of inflammatory process of atherogenesis. In nephrotic syndrome, hypoalbuminemia contributes to increase cardiovascular disease by elevating lysophosphatidylcholine [32]. Considering these previous reports, we postulate that hypoalbuminemia is closely associated with vascular atherosclerosis and cardiovascular events for which loss of nighttime BP decline is the crucial risk marker. Even though the range of serum albumin levels is narrow, Fung et al. [33] and Mehrotra et al. [34] indicated that a decrease by as little as $0.2 \mathrm{~g} / \mathrm{dL}$ increases cardiovascular risk and mortality. Thus, investigations so far indicate that serum albumin level alone should have potential influence on the circadian BP rhythm and consequently is a relevant predictor of cardiovascular disease.

\section{Limitations}

First, participants were recruited from patients referred to our outpatient clinic of internal medicine and this limits the generalizability of the findings. However, the subjects were not selected only on the basis of albuminuria, and hypertensive-naïve individuals were selected for the main studies. Therefore, selection bias and medication are unlikely to influence the findings. Second, the diabetic and nondiabetic groups differed in age. Thus comparison between the two groups requires caution. Nevertheless, our analysis has several strengths in comparing subgroups within the two groups. Third, due to the small numbers of subjects after stratification, we did not separate mildly hypertensive from normotensive patients based on baseline BP among the hypertensive-naïve subjects. However, an analysis showed that inclusion of the mildly hypertensive patients did not influence the ANOVA results (data not shown). Fourth, we regarded the nephrotic syndrome as a single entity, because although the syndrome consists of a diverse range of underlying renal histopathologies, there are no hemodynamic differences (unpublished data).

In conclusions, our study suggests that serum albumin level as well as urinary albumin excretion affect the circadian BP rhythm in early stage non-diabetic CKD. However, to confirm our hypothesis, a larger number of subjects with massive albuminuria or hypoalbuminemia of various non-renal causes should be evaluated.

\section{References}

1. Lurbe E, Redon J, Kesani A, Pascual JM, Tacons J, et al. (2002) Increase in nocturnal blood pressure and progression to microalbuminuria in type 1 diabetes. N Engl J Med 347: 797-805

2. Nielsen FS, Rossing P, Bang LE, Svendsen TL, Gall MA, et al. (1995) On the mechanisms of blunted nocturnal decline in arterial blood pressure in NIDDM patients with diabetic nephropathy. Diabetes 44: 783-789.

3. Poulsen PL, Ebbehøj E, Hansen KW, Mogensen CE (1999) Characteristics and prognosis of normoalbuminuric type 1 diabetic patients. Diabetes Care 22 B72-75

4. Pecis M, Azevedo MJ, Moraes RS, Ferlin EL, Gross JL (2000) Autonomic dysfunction and urinary albumin excretion rate are associated with an abnormal blood pressure pattern in normotensive normoalbuminuric type 1 diabetic patients. Diabetes care 23: 989-993.

5. Spallone V, Maiello MR, Cicconetti E, Pannone A, Barini A, et al. (2001) Factors determining the 24-h blood pressure profile in normotensive patients with type 1 and type 2 diabetes. J Hum Hypertens 15: 239-246.

6. Bianchi S, Bigazzi R, Baldari G, Sgherri G, Campese VM (1994) Diurnal variations of blood pressure and microalbuminuria in essential hypertension. Am J Hypertens 7: 23-29.

7. Kohara K, Nishida W, Maguchi M, Hiwada K (1995) Autonomic nervous function in non-dipper essential hypertensive subjects. Evaluation by power spectra analysis of heart rate variability. Hypertension 26: 808-814.

8. Bauduceau B, Genès N, Chamontin B, Vaur L, Renault M, et al. (1998) Ambulatory blood pressure and urinary albumin excretion in diabetic (noninsulin-dependent and insulin-dependent) hypertensive patients: relationships at baseline and after treatment by the angiotensin converting enzyme inhibitor trandolapril. Am J Hypertens 11: 1065-1073.

9. Rossing K, Christensen PK, Andersen S, Hovind P, Hansen HP, et al. (2003) Comparative effects of Irbesartan on ambulatory and office blood pressure: a substudy of ambulatory blood pressure from the Irbesartan in Patients with Type 2 Diabetes and Microalbuminuria study. Diabetes Care 26: 569-574.

10. Lacourcière Y, Bélanger A, Godin C, Hallé JP, Ross S, et al. (2000) Long-term comparison of losartan and enalapril on kidney function in hypertensive type 2 diabetics with early nephropathy. Kidney Int 58: 762-769.

11. Yasuda G, Ando D, Hirawa N, Umemura S, Tochikubo O (2005) Effects of losartan and amlodipine on urinary albumin excretion and ambulatory blood pressure in hypertensive type 2 diabetic patients with overt nephropathy. Diabetes Care 28: 1862-1868.

12. Yasuda G, Hasegawa K, Kuji T, Ogawa N, Shimura G, et al. (2005) Effects of doxazosin on ambulatory blood pressure and sympathetic nervous activity in hypertensive Type 2 diabetic patients with overt nephropathy. Diabet Med 22 1394-1400.

13. Boulatov VA, Stenehjem A, Os I (2001) Association between albumin:creatinine ratio and 24-hour ambulatory blood pressure in essential hypertension. Am J Hypertens 14: 338-344.

14. Arnlöv J, Evans JC, Meigs JB, Wang TJ, Fox CS, et al. (2005) Low-grade albuminuria and incidence of cardiovascular disease events in nonhypertensive and nondiabetic individuals: the Framingham Heart Study. Circulation 112: 969-975.

15. Jha V, Ganguli A, Saha TK, Kohli HS, Sud K, et al. (2007) A randomized, controlled trial of steroids and cyclophosphamide in adults with nephrotic syndrome caused by idiopathic membranous nephropathy. J Am Soc Nephrol 18: $1899-1904$.

16. Equiluz-Bruck S, Schnack C, Kopp HP, Schernthaner G (1996) Nondipping of nocturnal blood pressure is related to urinary albumin excretion rate in patients with type 2 diabetes mellitus. Am J Hypertens 9: 1139-1143.

17. Pontremoli R, Nicolella C, Viazzi F, Ravera M, Sofia A, et al. (1998) Microalbuminuria is an early marker of target organ damage in essential hypertension. Am J Hypertens 11: 430-438.

18. Berrut G, Bouhanick B, Fabbri P, Guilloteau G, Lalanne P, et al. (1996) Loss of the nocturnal decline in blood pressure in subjects with essential hypertension and microalbuminuria. Blood Press Monit 1: 469-473.

19. Diercks GF, Stroes ES, van Boven AJ, van Roon AM, Hillege HL, et al. (2002) Urinary albumin excretion is related to cardiovascular risk indicators, not to flow-mediated vasodilation, in apparently healthy subjects. Atherosclerosis 163: 121-126.

20. Gerber LM, Schwartz JE, Cedeno-Mero C, Warren K, Pickering TG (2001) 
Citation: Yasuda G, Ando D, Hirawa N (2014) Hypoalbuminemia and Hyperalbuminuria as Major Factors Regulating Circadian Blood Pressure Rhythm in Normal Subjects and Non-diabetic Patients with Early Stages of Chronic Kidney Disease. J Hypertens 3: 142. doi:10.4172/21671095.1000142

Association of urinary albumin concentration with casual and ambulatory blood pressure: a similar relationship in normotensive and hypertensive subjects. Blood Press Monit 6: 245-251.

21. Brantsma AH, Bakker SJ, de Zeeuw D, de Jong PE, Gansevoort RT (2006) Urinary albumin excretion as a predictor of the development of hypertension in the general population. J Am Soc Nephrol 17: 331-335

22. Lengyel Z, Rosivall L, Németh C, Tóth LK, Nagy V, et al. (2003) Diurnal blood pressure pattern may predict the increase of urinary albumin excretion in normotensive normoalbuminuric type 1 diabetes mellitus patients. Diabetes Res Clin Pract 62: 159-167.

23. Klausen K, Borch-Johnsen K, Feldt-Rasmussen B, Jensen G, Clausen P, et al. (2004) Very low levels of microalbuminuria are associated with increased risk of coronary heart disease and death independently of renal function, hypertension, and diabetes. Circulation 110: 32-35.

24. Hoshide S, Kario K, Hoshide Y, Umeda Y, Hashimoto T, et al. (2003) Associations between nondipping of nocturnal blood pressure decrease and cardiovascular target organ damage in strictly selected community-dwelling normotensives. Am J Hypertens 16: 434-438.

25. Soylu A, Yazici M, Duzenli MA, Tokac M, Ozdemir K, et al. (2009) Relation between abnormalities in circadian blood pressure rhythm and target organ damage in normotensives. Circ J 73: 899-904.

26. Clausen $P$, Jensen JS, Borch-Johnsen K, Jensen G, Feldt-Rasmussen B (1998) Ambulatory blood pressure and urinary albumin excretion in clinically healthy subjects. Hypertension 32: 71-77.
27. Koomans HA, Braam B, Geers AB, Roos JC, Dorhout Mees EJ (1986) The importance of plasma protein for blood volume and blood pressure homeostasis. Kidney Int 30: 730-735.

28. Vande Walle JG, Donckerwolcke RA (2001) Pathogenesis of edema formation in the nephrotic syndrome. Pediatr Nephrol 16: 283-293.

29. Agarwal R, Andersen MJ (2005) Correlates of systolic hypertension in patients with chronic kidney disease. Hypertension 46: 514-520.

30. Yokoyama H, Tomonaga O, Hirayama M, Ishii A, Takeda M, et al. (1997) Predictors of the progression of diabetic nephropathy and the beneficial effect of angiotensin-converting enzyme inhibitors in NIDDM patients. Diabetologia 40: 405-411

31. Zimmermann J, Herrlinger S, Pruy A, Metzger T, Wanner C (1999) Inflammation enhances cardiovascular risk and mortality in hemodialysis patients. Kidney Int 55: 648-658.

32. Vuong TD, Stroes ES, Willekes-Koolschijn N, Rabelink TJ, Koomans HA, et al. (1999) Hypoalbuminemia increases lysophosphatidylcholine in low-density lipoprotein of normocholesterolemic subjects. Kidney Int 55: 1005-1010.

33. Fung F, Sherrard DJ, Gillen DL, Wong C, Kestenbaum B, et al. (2002) Increased risk for cardiovascular mortality among malnourished end-stage renal disease patients. Am J Kidney Dis 40: 307-314.

34. Mehrotra R, Duong U, Jiwakanon S, Kovesdy CP, Moran J, et al. (2011) Serum albumin as a predictor of mortality in peritoneal dialysis: comparisons with hemodialysis. Am J Kidney Dis 58: 418-428. 